\title{
Analyzing the Performance of Various Corporate Networks using Multi-Protocol Label Switching Technology
}

\author{
Manav Soneja \\ School of Electronics Engineering \\ VIT University, Vellore \\ Tamil Nadu, India
}

\author{
Prof. Ravi Kumar C V \\ School of Electronics Engineering \\ VIT University, Vellore \\ Tamil Nadu, India
}

\begin{abstract}
Mulit-protocol label switching has been a building block in providing WAN connectivity. Traffic from multiple customers can travel over the service provider's shared MPLS network, making it a VPN service. MPLS VPN provides efficiency for very large networks. It lends scalability, security, and high quality of service to a network. This project encompasses the behavior of a MPLS and a Non-MPLS network when various services like web, VoIP, DNS are deployed on it. Our work emphasis on the way MPLS is capable of handling heavy traffic in large scale networks along with better performance based on the parameters -jitter, latency and round trip time.
\end{abstract}

\section{Keywords-MPLS; VPN;BGP; OSPF;RTT; EIGRP}

\section{INTRODUCTION}

Mulit-protocol label switching (MPLS) has been a crucial technique for Wide Area Network (WAN) connectivity. Large chunks of data, heavy traffic from multiple customers can travel over the service provider's shared MPLS network, making it a Virtual Private Network (VPN) service. MPLS VPN provides efficiency for very large networks. MPLS is used to provide scalability, security and high quality of service to a network. This project encompasses the behavior of a MPLS and a Non-MPLS network when various waveform codecs like g711alaw, g711ulaw and g729a are deployed on it. MPLS VPN network provides path protection and it proved by implementing the scenario in GNS3 software. This implementation is incorporated with IP SLA to analyze the latency, jitters and round trip time corresponding to the application deployed in the network. We use IP SLA to generate traffic. It is used to generate various types of services such as http, DNS, TCP, UDP echo etc. Therefore, IP SLA is used to generate waveform codecs.

MPLS protocol is incorporated to speed up and shape the way the traffic flows. It works on short path labels to direct the data through dedicated paths towards the destination, in order to transmit information from one network node to another node. It is faster as it doesn't make use of the long network addresses, therefore, avoiding complex lookups in the routing table. This technology is very popular among service providers for fast and reliable delivery of data traffic between Provider Edge to Customer Edge. Cisco IOS IP SLAs can help generate all types of traffic and can also continuously collect data about such things as response times, latency, jitter and packet loss. This not only provides the network administrator with baseline information about network performance, it also helps the administrator to analyze Quality of Service (QoS) levels and quickly identify the cause of a problem if performance levels deteriorate. The Network Management System (NMS), can be integrated to capture visual alerts in threshold violations in real-time. Tools like Wireshark and Solar Winds can be integrated with GNS3 emulator to capture and analyze real time traffic.

In our work, we implement MPLS technology to measure the network performance based on parameters such as jitter, latency, and round trip time (RTT). The result will be compared to the network that uses the regular routing switching technique. We analyze the network performance for two topologies-a smaller one and the other slightly larger. We do this to comprehend the behavior of MPLS in large scale network in contrast to the Non-MPLS technique.

\section{ROUTING}

Routing refers to determining the routes or paths that data information packets follow in order to reach a particular destination. Routing can be applied to large scale internet networks as well, over $3 \mathrm{G}$ or $4 \mathrm{G}$ networks that are used for telecom purpose or other digital communication. Routing can also take place within proprietary networks.

\section{A. Open Shortest Path First (OSPF)}

It is a link-state routing protocol. In a link-state routing protocol, the routers exchange topology information with their nearest neighbors. The topology information is flooded throughout the Autonomous System (AS), so that every router within the AS has a complete picture of the topology of the AS. This picture is then used to calculate end-to-end paths through the AS, normally using a variant of the Dijkstra algorithm. In a link-state routing protocol, the next hop address for a data packet is determined by choosing the best end-to-end path to the eventual destination. OSPF supports large networks, and has a very fast convergence time. It uses 'cost' metric which is derived from the bandwidth of the interface used. Default Administrative Distance (AD) for OSPF is 110 .

\section{B. Enhanced Interior Gateway Routing Protocol (EIGRP)}

EIGRP is a distance vector routing protocol. In a distance vector routing protocol, a router stores copies of all its 
neighbors' routing tables so that it can quickly adapt to alternate routes. If no appropriate route exists, EIGRP queries its neighbors to discover an alternate route. This process keeps running until an alternate route is found. Unlike some earlier routing protocols that would send an entire table to neighboring routers when one routing table entry changed, EIGRP notifies the neighbors of only the specific change in the table. EIGRP uses the Diffusing-Update Algorithm (DUAL) developed at SRI International. EIGRP uses bandwidth and delay of the links to calculate the metric. Default Administrative Distance (AD) for EIGRP is 90. It automatically performs equal cost load balancing on up to a maximum of 16 paths.

\section{Border Gateway Protocol (BGP):}

BGP is the only Exterior Gateway Protocol (EGP), unlike OSPF, RIP, EIGRP which are Interior Gateway Protocols (IGPs). BGP controls routing on internet. Rather than sharing information and making decisions on a physical hop by physical hop basis, BGP works on Autonomous System (AS) by AS analysis. An AS is a portion of a large network (such as internet) which is under a single administrative control. BGP routers are not aware of the individual physical hops traffic takes as it traverses another AS. They see an overall AS as an individual hop. This makes the solution more scalable, this where into plays a crucial role in MPLS networks. BGP is a vector routing protocol, it chooses the path with the shortest AS path. Default AD for BGP is 20. AD is used to choose between multiple paths learned via different routing protocols.

\section{Multi-Protocol Label Switching (MPLS)}

Multiprotocol Label Switching (MPLS) is a kind of of network data and services traffic managing technique which delivers data from one node to the next node using short path labels instead of wasting time using long and complex network router lookups in a routing table. MPLS is best considered as a middleman protocol between Layer 2 and Layers 3 in the OSI model. A MPLS network acts like a giant switch.

In MPLS, packets are directed through the network based on an assigned label. The label is linked with a predetermined path through the network, which allows a higher level of control than in packet-switched networks. As packets travel through the MPLS network, their labels are switched or swapped. The data packet enters the MPLS network at a Label Switched Router (LSR), where it is is examined, classified and given an appropriate label, and forwarded to the next hop in the pre-set Label Switched Path (LSP). As the packet travels that path, each router on the path uses the label - not other information, such as the IP header - to make the forwarding decision that keeps the packet moving along the LSP.

\section{E. IP Service Level Agreement (IP SLA)}

IP SLA is an active method used to monitor and reliably report on network performance. Here "active," refers to the fact that IP SLA will generate and actively monitor traffic continuously across the network. An IP SLA Router is capable of generating traffic and reporting and estimating parameters on it in real time. IP SLA can be configured in such a way that it can report on statistics such as:

- Jitter

- Response time

- Packet loss

- $\quad$ Voice Quality Scoring (MOS)

- Connectivity

- Server or website responses and downtime

- Delay

\section{F. Packet Forwarding Traditional IP Versus network MPLS}

IP uses hop-by-hop destination-only forwarding paradigm. In traditional IP Packet forwarding, each router needs to look up the data packet's destination IP address in the IP routing table in order to decide the next-hop router for the packet. MPLS uses a variety of routing protocols to establish Label Switched Paths (LSP) across the network.

Label Switched Paths provides the ideal route to the destination, they are similar to Asynchronous Transfer Mode (ATM) and Permanent Virtual Circuit (PVC), but the difference being that they are unidirectional and they can merge. MPLS networks incorporate a protocol i.e., Label Distribution Protocol (LDP). LDP builds the LSPs based on the IP routing table, making an MPLS network automatically functionally equivalent to a pure IP network.

Once the set of LSPs has been fixed, it is used to forward IP packets: the first router of the MPLS backbone known as as the ingress router inserts a label (or a stack of them) at the beginning of the IP header and forwards the packet. All the subsequent Label Switch Routers (LSR) ignore the IP headers and perform packet forwarding based on the labels attached to them. Finally, the last router of the MPLS network called the egress router removes the label and forwards the original IP packet towards its final destination. In theory, MPLS forwarding proves to be faster than IP forwarding (due to simpler label lookup).

\section{METHODOLOGY}

To support this research, it takes a design scenario so that it can conduct performance comparisons between the Non MPLS technology (routing switching) and MPLS technology that will be used. The design scenario use the same topology model but using different technologies (Routing Switching and MPLS). In this study we use sample network model, we have to do the configuration or settings on each device that will be used. The configurations given to the device depend on the model of scenario testing to be performed. The system topology is designed using emulator software GNS-3. The network model on GNS-3 will be made similar to the model that will be used in laboratory research.

The analytical method used is quantitative analysis method in which the measurement to obtain a value for the parameter tested from each study. The data which used to measure are ICMP echoes, and waveform codecs like G711alaw, G711ulaw and G729a packets. These data will be sent across the network and all the parameter in the header 
will be measured using the tool: IP SLA. Basically, we will perform experimental with the following steps:

- Perform g711ulaw, g711alaw and g729a data transmission using MPLS technology.

- Perform g711ulaw, g711alaw, g729a data transmission technology using Routing Switching.

- Conduct an evaluation for each routing table of each technology as a reference for the considering the performance of the router in finding the best path.

- Do trace the network when the data delivery process from source to destination.

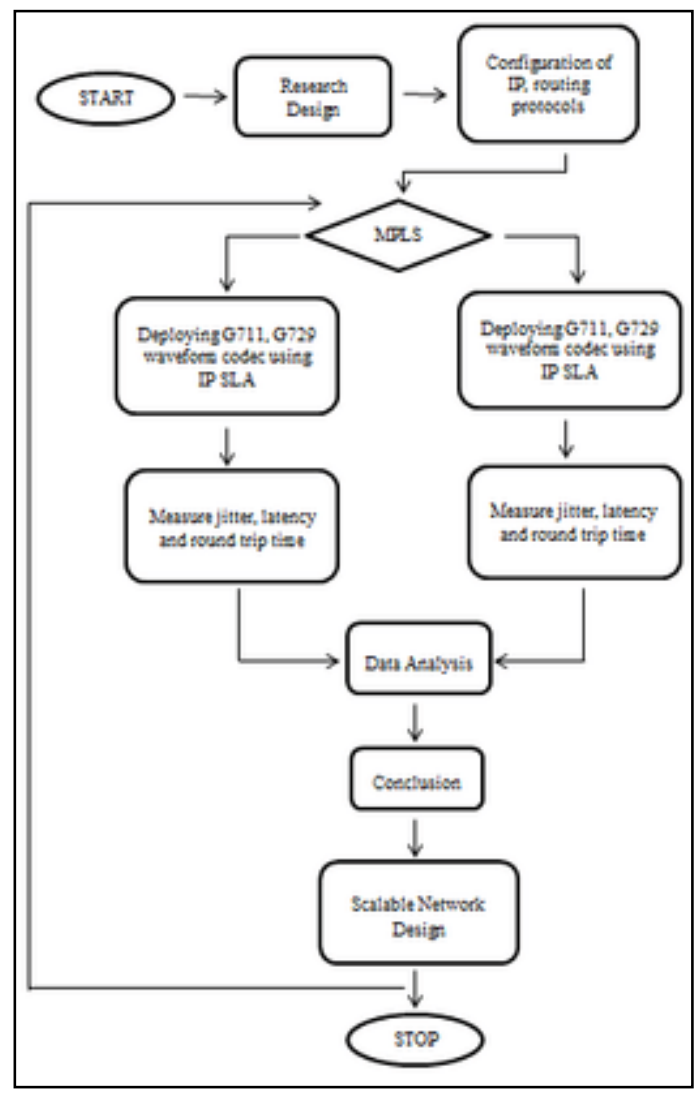

Fig. 1 Block Diagram

The complete steps of the methodology can be seen in figure 1. In figure 1 , we can see once we are done with configuration of IP on the router $\mathrm{PE}$, then we made comparison between MPLS and Non-MPLS. We took the data from IP SLA to measure performance of MPLS and the data to measure performance Non-MPLS was taken from routing table in routing switching. We repeat this process for a larger network topology to analyze the performance of services in a larger network.

We measure latency, round trip time and jitter for both of method, MPLS and non MPLS. Finally we compared both of data and made some data analyzing on those data. The hardware that we used in this research are:
- Cisco 3600 Series Router:

Cisco 3600 series provides multifunction platform, combining dial access, routing, and LAN=to-LAN services along with multi-service integration of multimedia as well as data in the same device. The Cisco 3600 series is fully supported by Cisco IOS software, which includes analog and digital voice capability, ATM access with T1/E1 IMA or OC3 interfaces, dial-up connectivity, LAN-to-LAN routing, data and access security, WAN optimization, and multimedia features. The Cisco 3660 has six network module slots. 3600 series routers are standard routers, consisting of high-speed console and auxiliary ports. The 3600 series consists of Cisco 3620, Cisco 3640, Cisco 3631 and Cisco 3600 Routers. It is capable of supporting DRAM or SRAM, Non-volatile RAM (NVRAM), flash memory and EPROM-based memory.

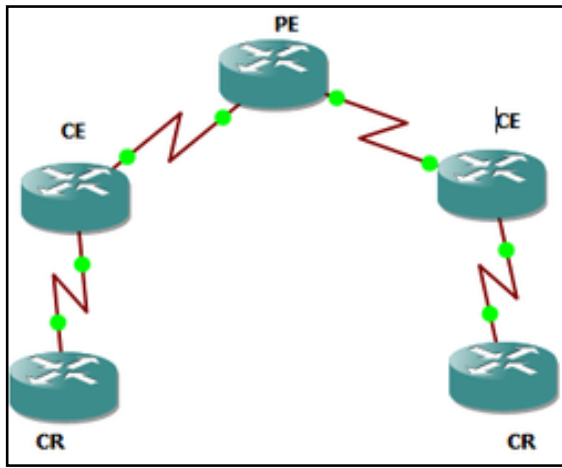

Fig. 2 Network Topology 1

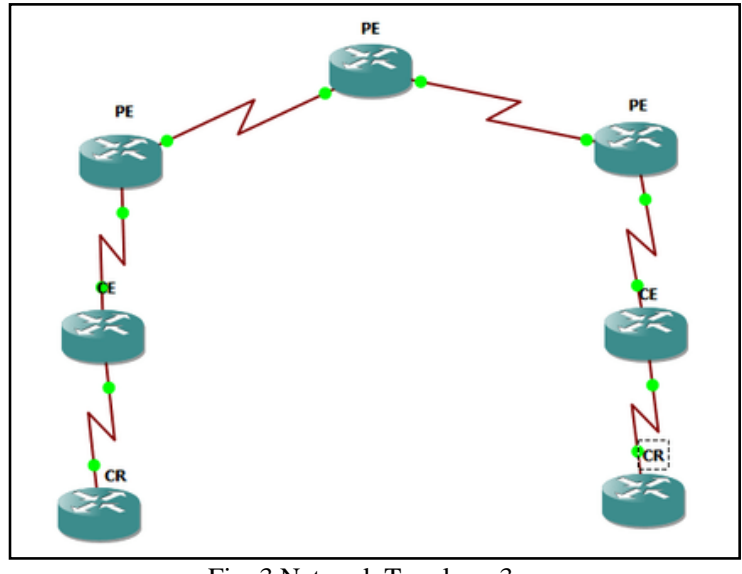

Fig. 3 Network Topology 3

Fig. 2 and Fig. 3 are the topologies followed by us in our research work to analyze the performance of an MPLS network. Fig. 3 is used to highlight the performance of MPLS in large scale internet networks.

Fig. 2 topology has three service provider routers and two customer end routers, while Fig. 3 topology has 5 service provider routers and two customer end routers. Our work focuses on the capability of MPLS to handle larger networks.

The traffic will send using MPLS and non-MPLS technologies than the comparison will be made with quantitative approach against of throughput, jitter and packet loss. Routers that become Provider Edge (PE) will check the types of traffic passed during testing by either MPLS technology or non-MPLS (Routing Switching). G711alaw, G711ulaw and G729a waveform codec services are enabled 
using IP SLA in GNS3 software to determine the value of round trip time, latency and jitter.

\section{RESULTS}

This research was conducted the testing using technology Switching Routing and MPLS technologies. The data of results of this study was found by capturing the traffic on both the client side. In each client was used software IP SLA as a traffic generator that sends traffic between clients so that we get the output of the inter-client traffic. There are several scenarios that will be carried on as follow:

- Deploy waveform codec and ICMP echo packets in network between clients to collect data about latency, round trip time and jitter.

- Deploy waveform codec and ICMP echo packets in network between clients using MPLS technology to collect data about latency, round trip time and jitter.

- Analyzing the performance of the services and comparing the results for MPLS and Non-MPLS technology.

- Increasing the scalability of the network, and repeating the above process to analyze the behavior of services in large scale network.

\begin{tabular}{|c|c|c|c|c|c|c|}
\hline \multirow[t]{2}{*}{ Parameter } & \multicolumn{2}{|c|}{ G711alaw } & \multicolumn{2}{|c|}{ G711ulaw } & \multicolumn{2}{|l|}{ G729a } \\
\hline & $\begin{array}{l}\text { Topol } \\
\text { ogy } 1 \\
\end{array}$ & $\begin{array}{l}\text { Topolo } \\
\text { gy } 2\end{array}$ & $\begin{array}{l}\text { Topol } \\
\text { ogy } 1\end{array}$ & $\begin{array}{l}\text { Topolo } \\
\text { gy } 2\end{array}$ & $\begin{array}{l}\text { Topol } \\
\text { ogy } 1 \\
\end{array}$ & $\begin{array}{l}\text { Topolo } \\
\text { gy } 2\end{array}$ \\
\hline $\begin{array}{l}\text { RTT } \\
\text { (min./avg./ } \\
\text { max.) } \\
(\mathrm{ms})\end{array}$ & $\begin{array}{l}79 / 13 \\
3 / 182\end{array}$ & $\begin{array}{l}357 / 45 \\
3 / 506\end{array}$ & $\begin{array}{l}73 / 14 \\
1 / 198\end{array}$ & $\begin{array}{l}309 / 47 \\
1 / 524\end{array}$ & $\begin{array}{l}81 / 14 \\
0 / 210\end{array}$ & $\begin{array}{l}371 / 45 \\
9 / 528\end{array}$ \\
\hline $\begin{array}{l}\text { Latency S } \\
\text { to } \mathrm{D} \\
\text { (min./avg./ } \\
\text { max.) } \\
(\mathrm{ms})\end{array}$ & $\begin{array}{l}2 / 32 / 6 \\
5\end{array}$ & $0 / 0 / 0$ & $\begin{array}{l}8 / 39 / 7 \\
3\end{array}$ & $0 / 0 / 0$ & $\begin{array}{l}16 / 51 \\
/ 88\end{array}$ & 0/0/0 \\
\hline $\begin{array}{l}\text { Jitter } S \text { to } \\
\text { D } \\
\text { (min./avg./ } \\
\text { max.) } \\
\text { (ms) }\end{array}$ & $\begin{array}{l}3 / 13 / 2 \\
3\end{array}$ & $\begin{array}{l}5 / 41 / 2 \\
17\end{array}$ & $\begin{array}{l}2 / 15 / 3 \\
2\end{array}$ & $\begin{array}{l}0 / 40 / 2 \\
16\end{array}$ & $\begin{array}{l}3 / 15 / \\
27\end{array}$ & $\begin{array}{l}8 / 46 / 21 \\
6\end{array}$ \\
\hline
\end{tabular}

Table 1 Result for MPLS Network for 10 Packets

\begin{tabular}{|l|l|l|l|l|l|l|}
\hline & \multicolumn{2}{|l|}{} & \multicolumn{2}{l|}{ G711ulaw } & \multicolumn{2}{l|}{ G729a } \\
\hline & $\begin{array}{l}\text { Topolo } \\
\text { gy 1 }\end{array}$ & $\begin{array}{l}\text { Topol } \\
\text { ogy 2 }\end{array}$ & $\begin{array}{l}\text { Topol } \\
\text { ogy 1 }\end{array}$ & $\begin{array}{l}\text { Topol } \\
\text { ogy 2 }\end{array}$ & $\begin{array}{l}\text { Topol } \\
\text { ogy 1 }\end{array}$ & $\begin{array}{l}\text { Topol } \\
\text { ogy 2 }\end{array}$ \\
\hline $\begin{array}{l}\text { RTT } \\
\text { (min./avg. } \\
\text { /max.) } \\
\text { (ms) }\end{array}$ & $\begin{array}{l}41 / 141 / \\
184\end{array}$ & $\begin{array}{l}434 / 63 \\
1 / 693\end{array}$ & $\begin{array}{l}30 / 123 \\
/ 168\end{array}$ & $\begin{array}{l}156 / 20 \\
7 / 277\end{array}$ & $\begin{array}{l}319 / 66 \\
3 / 840\end{array}$ & $\begin{array}{l}153 / 22 \\
7 / 268\end{array}$ \\
\hline $\begin{array}{l}\text { Latency S } \\
\text { to D } \\
\text { (min./avg. } \\
\text { /max.) } \\
\text { (ms) }\end{array}$ & $0 / 0 / 0$ & $0 / 0 / 0$ & $0 / 0 / 0$ & $0 / 0 / 0$ & $0 / 0 / 0$ & $0 / 0 / 0$ \\
\hline $\begin{array}{l}\text { Jitter S to } \\
\text { D } \\
\text { (min./avg. } \\
\text { /max.) } \\
\text { (ms) }\end{array}$ & $1 / 14 / 32$ & $2 / 40 / 1$ & $0 / 13 / 2$ & $0 / 18 / 5$ & $0 / 11 / 1$ & $0 / 14 / .4$ \\
57 & 3 & 6 & 12 & 1 \\
\hline
\end{tabular}

Table 2 Result for Non-MPLS Network for 10 Packets

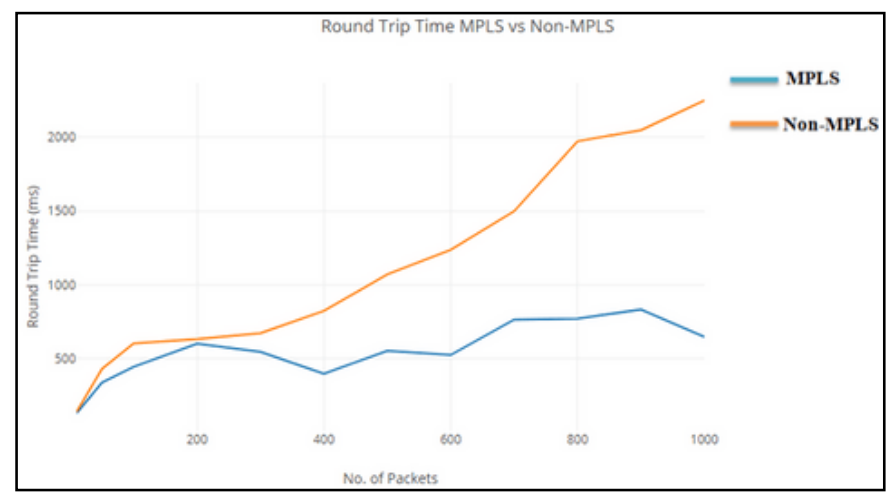

Fig. 4 Round Trip Time MPLS vs Non-MPLS Topology 1

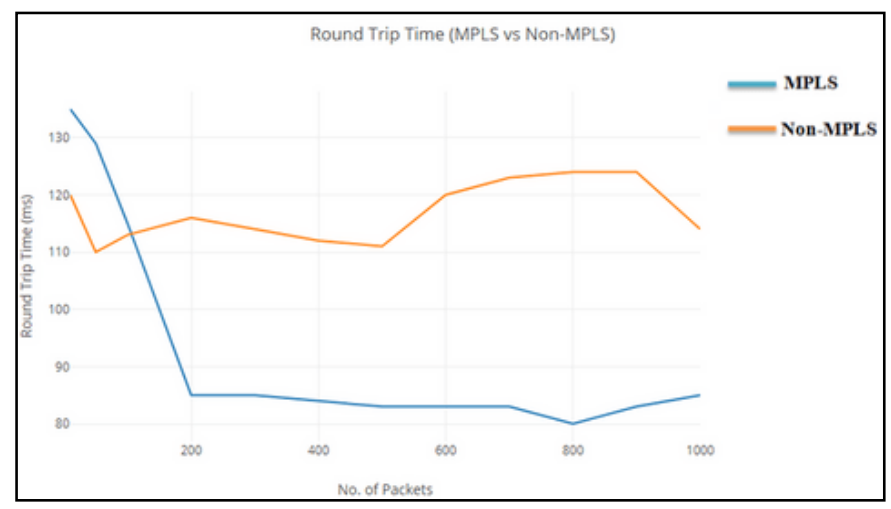

Fig. 5 Round Trip Time MPLS vs Non-MPLS Topology 2

\section{A. Round Trip Time}

Round Trip Time (RTT) estimates the time in milliseconds (ms) it takes for a network request to go from the starting point to the destination and back again to the starting point. RTT is an important metric to determine the health of a network connection in a local area network or large scale internet network. Reducing the round trip time is the job of a network administrator. 
Fig. 4 is the result of comparing the round trip time in a MPLS and a Non-MPLS network. The graph showcases the change in round trip time in accordance with the no. of packets. Clearly, the round trip time for a Non-MPLS network escalates with the increase in no. of packets when compared to a MPLS network. Round trip time average for g711alaw service under MPLS network is $547 \mathrm{~ms}$ whereas for a Non MPLS network it is $1115 \mathrm{~ms}$.

Fig. 5 is the result of comparing the RTT of topology 2 for MPLS and Non-MPLS network. Average RTT for MPLS is $94.16 \mathrm{~ms}$ and for Non-MPLS is $1116.75 \mathrm{~ms}$. MPLS networks are capable of improving the performance of large scale networks. Comparing Fig. 4 and Fig. 5, it is clearly evident how MPLS is able to sustain the level of high performance even for large networks. For Non-MPLS the performance gets compromised in both the cases, when no. of packets being transmitted is increased as well as when the network is made larger.

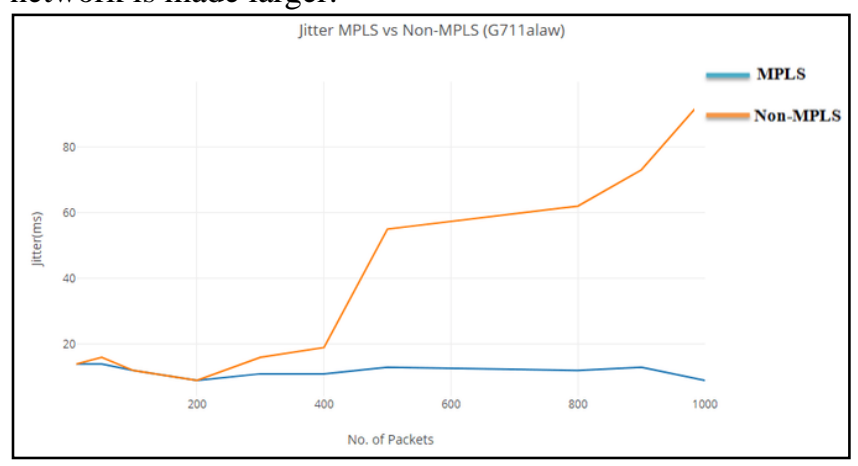

Fig. 6 Jitter MPLS vs Non-MPLS Topology 1

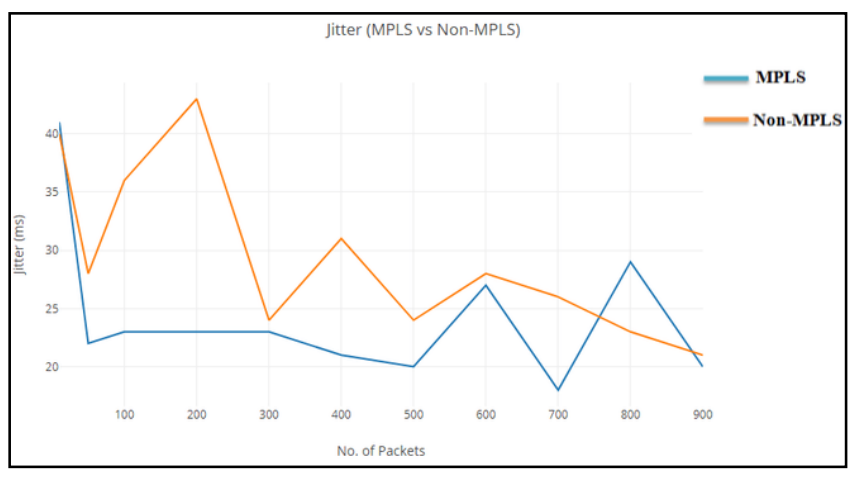

Fig. 7 Jitter MPLS vs Non-MPLS Topology 2

\section{B. Jitter}

Fig. 6 highlights the contrast between the jitter when services deployed in a MPLS and a Non-MPLS network. Jitter is basically the difference in the delay of packets. Jitter is popular in the transmission of voice packets in our case the transmission of g711 and g729 waveform codecs. Jitter refers to the delay in receiving a voice packet. This delay effects the transmission of voice quality and voice data. Fig. 6 shows how the jitter increases exponentially in a Non-MPLS network. Average jitter for g711alaw codec in case of an NonMPLS network is $33.25 \mathrm{~ms}$, while on the other hand it is 11.91 for a MPLS network.

Fig. 7 is comparison between the MPLS and Non-MPLS jitter of topology 2. Overall jitter for MPLS is less as compared to Non-MPLS which eventually lowers. Average jitter for Non-MPLS is $29.2 \mathrm{~ms}$ while MPLS is $29 \mathrm{~ms}$.

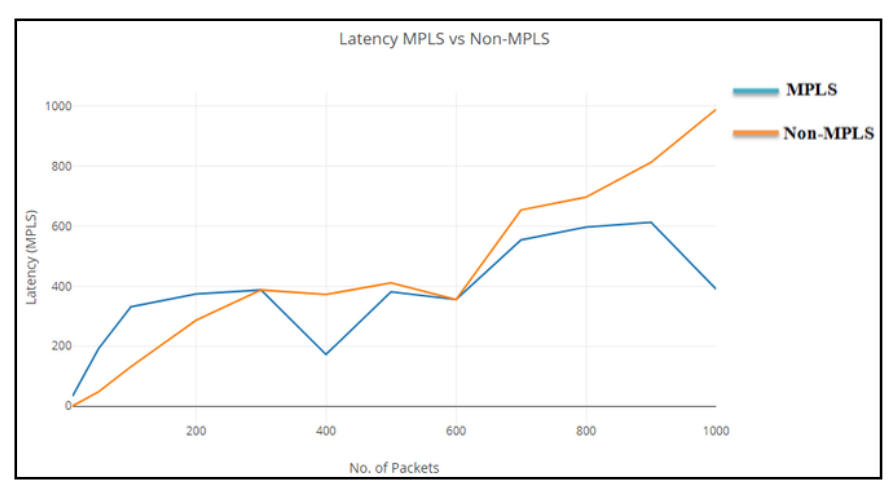

Fig. 8 Latency MPLS vs Non-MPLS Topology 1

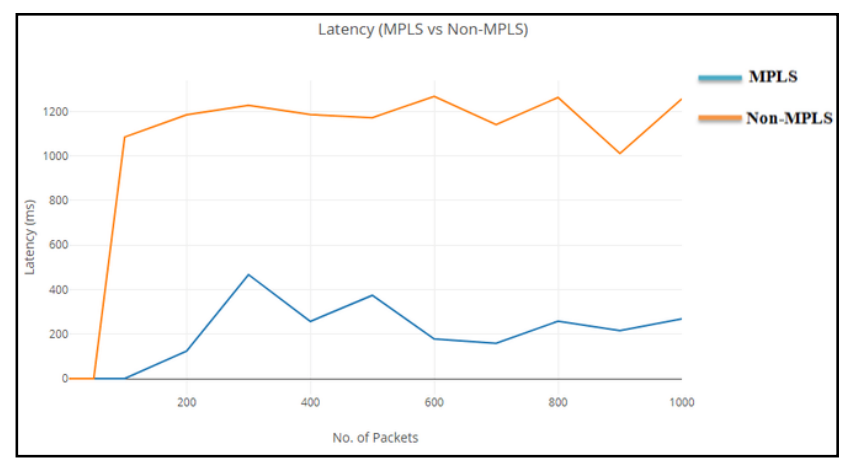

Fig. 9 Latency MPLS vs Non-MPLS Topology 2

\section{Latency}

Fig. 8 tells about the latency in a MPLS and a Non-MPLS network. Network latency refers to the small delays that occur over a network during data communication. According to our results, average latency for a MPLS network is $364.75 \mathrm{~ms}$ and $428.58 \mathrm{~ms}$ for a Non-MPLS network. High latency is creates bottlenecks in a network communication. High latency prevents the data from taking full advantage of the bandwidth of the transmission medium.

Fig. 9 draws a contrast between the latency of MPLS and Non-MPLS technique based on topology 2. It is evident that MPLS based technology has negligible latency when compared to Non-MPLS. Average latency for MPLS is $192.16 \mathrm{~ms}$ and average latency for Non-MPLS is $983 \mathrm{~ms}$.

\section{CONCLUSION}

Based on the results obtained from testing the network with MPLS and Non-MPLS technology we can conclude the following things:

- MPLS technology is more promising as it returns better performance statistics. The quality of services determined from three parameters latency, round trip time and jitter is high in MPLS enabled networks when compared to nonMPLS networks.

- Large scale internet networks are also best supported by MPLS networks. MPLS networks assure high stability under heavy traffic conditions for large scale networks. Non-MPLS networks showcase steep decline in the quality of services when exposed to heavy traffic. 


\section{REFERENCES}

[1] Benfano Soewito, Benfano Soewito, Sayed Afdhal, Anna Antonyova 2017. Analysis of Quality of Network Using MPLS and NonMPLS., International Seminar on Intelligent Technology and Application

[2] Rashed Qayoom Shawl, Rukhsana Thaker, Er. JAsvinder Singh 2014. A Review: Multi Protocol Label Switching, International Journal of Engineering Research and Applications
[3] Gustavo Lopez, Eduardo Grampin. Scalability Testing of Legacy MPLS-based Virtual Private Networks

[4] Jitendra Joshi, Sonali Gupta, Priti Gupta, Nisha Singh Manjari Kumari 2013. Multi Protocol Label Switching with Quality of Service in High Speed Computer, Network, International Journal of Engineering Science and Innovative Technology (IJESIT)

[5] K. K. Nguyen, B. Jaumard. A Distributed and Scalable MPLS Architecture for Next Generation Routers 